\title{
Curiosity Enhances Incidental Object Encoding in 8-month-old Infants
}

\author{
Xiaoyun Chen ${ }^{1}$, Katherine E. Twomey ${ }^{2}$, and Gert Westermann ${ }^{1}$ \\ ${ }^{1}$ Department of Psychology, Lancaster University, UK
}

${ }^{2}$ Division of Human Communication, Development and Hearing, University of Manchester, UK

\begin{abstract}
Author Note
We have no known conflict of interest to disclose. The authors confirm that the data and its supplementary materials are available on the Open Science Framework: https://osf.io/7r2dt/?view_only=02d905fb5b1d44e4be7eecdc1d46eb38

Correspondence concerning this article should be addressed to Xiaoyun Chen, Dept. of Psychology, D37, Fylde College, LA1 4YF. Email: x.chen24@lancaster.ac.uk (X.Chen)

This work was supported by the ESRC International Centre for Language and Communicative Development (LuCiD) [ES/L008955/1, ES/S007113/1].
\end{abstract}




\section{Research Highlights}

- Curiosity induced by uncertainty enhances learning of unrelated information in 8month-old infants.

- 8-month-old infants do not seek resolution of curiosity over experiencing novelty.

- This is the first empirical study to explore how states of curiosity affect object encoding in infants and the role of curiosity resolution in this process.

- Our results suggest that curiosity increases the state of arousal, facilitating learning in infants.

- Overall, based on currently available curiosity research, we suggest curiosity changes in infants from a broader state of arousal to more goal-directed information seeking in older children and adults. 


\begin{abstract}
Recent research with adults indicates that curiosity induced by uncertainty enhances learning and memory outcomes and that the resolution of curiosity has a special role in curiositydriven learning. However, the role of curiosity-based learning in early development is unclear. Here we presented 8-month-old infants with a novel looking time procedure to explore: 1) whether uncertainty-induced curiosity enhances learning of incidental information; and 2) whether uncertainty-induced curiosity leads infants to seek uncertainty resolution over novelty. In Experiment 1, infants saw blurred images to induce curiosity (Curiosity sequence) or a clear image (Non-Curiosity sequence) followed by presentation of incidental objects. Despite looking equally to the incidental objects in both sequences, in a subsequent object recognition phase infants looked longer to incidental objects presented in the Non-Curiosity than in the Curiosity condition, indicating that curiosity induced by blurred pictures enhanced the processing of the incidental object, leading to a novelty preference for the incidental object shown in the Non-Curiosity condition. In Experiment 2, a blurred picture of a novel toy was first presented, followed by its corresponding clear picture paired with a clear picture of a new novel toy side-by-side. Infants showed no preference for either image, providing no evidence for a drive to resolve uncertainty. Overall, the current studies suggest curiosity has a broad attention-enhancing effect in infancy. Taking into account existing studies with older children and adults, we propose a developmental change in the function of curiosity, from this attentional enhancement to more goal-directed information seeking in order children and adults.
\end{abstract}

Keywords: curiosity, curiosity-driven learning, uncertainty, object encoding, memory enhancement, infancy 


\section{Curiosity Enhances Incidental Object Encoding in 8-month-old Infants}

Curiosity - the intrinsically motivated search for information - has a long history of research but has recently re-entered the focus of scientific investigation. Curiosity has been described as a drive evoked by events of complexity, uncertainty and novelty (Berlyne, 1954, $1960,1966)$ that promotes exploratory behaviours leading to knowledge acquisition or improved perception of the environment (Gruber et al., 2019; Loewenstein, 1994). Different theories have been put forward to explain how curiosity motivates exploratory behaviors, such as the drive to close a knowledge gap (Loewenstein, 1994), generation of predictions that are then evaluated (Gruber \& Ranganath, 2019), or reduction of experienced uncertainty (Berlyne, 1960; Berlyne \& Normore, 1972; Jepma et al., 2012). In experimental studies, the two most frequent paradigms used to induce curiosity are asking participants trivia questions and presenting them with blurred images.

Different studies have shown how curiosity can be elicited by uncertainty (Berlyne, 1954; Gruber et al., 2019; Kang et al., 2009; Loewenstein, 1994; Nicki, 1970; Kobayashi et al., 2019; van Lieshout et al., 2018). For example, in a seminal study, Nicki (1970) presented adult participants with a series of images with low, medium and high degrees of blur to induce uncertainty, followed by the option to press one of two keys, with one revealing the clear corresponding image of the blurred image and the other an unrelated clear image. Participants' key presses leading to the clear corresponding images increased across trials. Moreover, participants' ratings of the blurred images indicated that they showed highest subjective uncertainty (and consequently, highest degree of curiosity) when stimuli were at a medium level of blurredness. In related work, Jepma and colleagues (2012) presented participants with (intermediate-degree) blurred images and used neuroimaging to study the brain regions involved in processing this information. They found that first, participants selfreported high curiosity for the blurred images (mean 4.11 on a 1-5 scale), and second, that the 
blurred images activated brain regions associated with autonomic arousal and aversive experience. When the participants were subsequently shown clear versions of the blurred images, they showed activation of brain regions linked to reward processing. Together, these studies suggest that in adults, blurred images (especially when blurred at an intermediate degree) reliably elicit strong curiosity, that participants seek to resolve this uncertainty, and that this resolution is intrinsically rewarding.

A core question in research on curiosity has been whether it supports learning. Overall, results using trivia questions or blurred picture paradigms have shown that both adult and child participants recalled information better when they were curious about it than when not (Berlyne \& Normore, 1972; Fandakova \& Gruber, 2021; Gruber et al., 2014; Jepma et al., 2012; Kang et al., 2009), suggesting that higher curiosity levels enhance attention and facilitate task-relevant information encoding. More recently, research has begun to investigate the extent to which curiosity enhances learning more generally, that is, whether enhanced learning is restricted to the object of curiosity, or whether a state of curiosity more generally facilitates learning of information that is encountered in this state. This work so far has led to mixed results. In one study, Gruber and colleagues (2014) presented adults with a sequence of trivia questions and asked them to rate their level of curiosity about each question. Then, before the answer was revealed, a face image as task-irrelevant information was presented. An immediate and a one-day delayed recall test showed that participants' recall of not only the task-relevant information (trivia question answers) but also the taskirrelevant information (faces) encountered during a high state of curiosity was enhanced relative to information learned during a low state of curiosity. However, contrasting with these results, in a study with children and adolescents using a similar paradigm, Fandakova and Gruber (2021) found that higher states of curiosity only enhanced learning of taskrelevant but not of task-irrelevant information. 
A second question of interest concerns the role of uncertainty resolution in curiosity. As discussed above, theories of curiosity often see the resolution of uncertainty as the main objective of curiosity-driven exploration. Evidence from adults suggests that uncertainty resolution is indeed implicated in curiosity; for example, when curiosity was triggered by blurred images, participants preferred to see a resolution over a novel image (Nicki, 1970), and participants reported higher disappointment when they were provided with a novel image instead of a resolution image after viewing blurred images (Jepma et al., 2012).

Whereas the study of curiosity in adults has a relatively long tradition, only recently has research begun to address the role of curiosity and active exploration in infants' knowledge acquisition (Kidd \& Hayden, 2015; Oudeyer \& Smith, 2016; Poli et al., 2020; Smith et al., 2018; Twomey \& Westermann, 2018). This early work suggests that infants are curious learners who actively navigate and structure their own learning, allocating their attention to the resources that allow them to maximize information gain to learn rapidly (Poli et al., 2020). This work has suggested intrinsically motivated exploration as a powerful mechanism to drive infants' learning and cognitive development, characterizing infants as active explorers instead of mere recipients of environmental information.

However, the mechanisms and effects of curiosity on learning and exploration in infancy are not well understood. Here we therefore addressed two questions in two experiments. First, guided by the idea that curiosity modulated by uncertainty facilitates learning in adults and older children (Fandakova \& Gruber, 2021; Gruber et al., 2014; Jepma et al., 2012), we asked whether in 8-month-old infants' curiosity supports learning globally beyond the specific object of curiosity. Second, we investigated whether infants, like adults, show a drive to resolve uncertainty by asking whether they prefer uncertainty resolution over novelty. 
Curiosity here is not conceptualized as a metacognitive awareness of 'not knowing' (e.g., as in Loewenstein, 1994), but as arousing a state of uncertainty that leads to further exploration (see e.g., Berlyne, 1954, 1960, 1966). It is well-known that animals and humans explore and seek information to reduce uncertainty (Berlyne, 1966; Bromberg-Martin \& Hikosaka, 2009; van Lieshout et al., 2018). Given that blurred stimuli induce strong curiosity in adults and since trivia questions are not suitable for use with infants, we used blurred stimuli as uncertain information to induce curiosity in the infant participants. Curiosity in older children and adults is assessed by self-report (e.g., answering the question 'how curious are you about this stimulus?') which is not possible with infants. While therefore we cannot be certain that the blurred images did induce curiosity in the infants, we believe so based on the literature showing that adults report high curiosity for such images, that blurred images represent uncertainty, and that uncertainty elicits exploratory behaviours even in non-human animals (Berlyne, 1966; Bromberg-Martin \& Hikosaka, 2009; Daddaoua et al., 2016). This uncertainty can be framed on a metacognitive level ("I don't know what this is.") but equally on a perceptual level (the information is hard to learn or represent and to link to existing knowledge), suggesting that metacognitive awareness of a knowledge gap is not a necessary precondition for curiosity to arise (Twomey \& Westermann, 2019).

Infants show considerable perceptual sensitivities and competencies from a very young age (Aslin \& Smith, 1988). They are sensitive to perceptual overlap between visual patterns (Fantz, 1958) and subtle changes in features of visual stimuli as early as the first three months of life (Quinn et al., 1993, 2001). Even newborn infants show certain levels of sensitivity to visual patterns differing only in configuration or form (Fantz \& Miranda, 1975). By 8 months of age, visual sensitivity develops rapidly to reach adult levels (Norcia \& Tyler, 1985; Skoczenski \& Norcia, 1998). We reasoned that on this basis, 8-month-old infants should be able to perceive the blurred stimuli. Moreover, given infants' sensitivity to 
perceptually overlapping stimuli (Quinn et al., 2001), we also presented the clear version of the blurred images to enable resolution of curiosity.

To investigate the breadth of learning facilitated by curiosity (Experiment 1), we presented infants with two sequences of images representing a Curiosity sequence and a NonCuriosity sequence. The Curiosity sequence consisted of a blurred image of a novel object to induce curiosity, followed by a different novel (clear) object image serving as incidental information not related to the object of curiosity, which was then followed by a clear version of the initial blurred picture. The Non-Curiosity sequence was similar to the Curiosity sequence except that the first image was always clear and not blurred. Immediately after infants saw these two sequences, we presented them with a preferential looking test in which the two incidental objects were shown side-by-side. We hypothesised that, if infants encoded the incidental objects encountered while in a state of curiosity (after seeing the blurred picture in the Curiosity sequence) or not (after seeing the clear picture in the Non-Curiosity sequence), they should show systematic preferences for either of the incidental objects at test.

To explore infants' resolution of uncertainty (Experiment 2), we presented them with a blurred image, followed by a preferential looking trial in which the clear corresponding image was paired with a new, clear image. We were interested in whether infants would show a looking preference to the clear corresponding image to resolve their curiosity over the novel image. Since Experiment 2 was substantially shorter than Experiment 1 (approximately one minute versus approximately seven minutes), we conducted Experiment 2 before Experiment 1 to maximise data quality for both experiments.

\section{Experiment 1: The Breath of Learning in Curiosity}

\subsection{Method}

\subsubsection{Participants}


Thirty-nine typically developing 8-month-old infants (Mage $=7$ months, 28 days; $S D$ $=9.68$ days; range 7 months 17 days -8 months, 16 days) and their caregivers were recruited from a database of parents who had indicated an interest in taking part in developmental research. This sample size was large enough to reach sufficient power (0.95) to detect an effect size of 0.7. All infants were born full-term and had no reported hearing or visual deficits. Caregivers' travel expenses were reimbursed, and infants were given a storybook as a gift for their participation. Informed consent was provided by the caregivers. The study was approved by the University's research ethics committee. Data from two infants were excluded for this experiment due to not contributing enough data for the final analysis (see Data processing and analysis below for exclusion criteria).

\subsubsection{Stimuli}

A total of 20 novel images were generated with Microsoft Paint 3D, which were then filtered with a 35-degree blur using a Gaussian filter in MATLAB (R2016b), resulting in 40 images with 20 blurred images and 20 corresponding clear images (see Figure 1 for sample images). All images were adapted to a similar rectangular size of approximately 450 by 350 pixels and placed on a grey background using Gimp (Version 2.10.8). No infant saw the same stimulus in more than one trial in this experiment.

Images were converted into videos with sound and animation effects at the onset of presentation for $700 \mathrm{~ms}$ in order to maintain infant engagement. Videos were produced on Microsoft PowerPoint by adding in-built animation effects with four different sound effects (cash register, laser, hammer and whoosh) and one animation effect (fly in from the top of the screen). Sound effects were counterbalanced across trials and participants. Additionally, 20 images of incidental, novel objects were selected from the NOUN database (Horst \& Hout, 2016). All images of novel objects were adapted into a similar rectangular size of approximately 450 by 350 pixels and placed on a grey background. 
Each pair of incidental objects selected from the NOUN database was matched based on a similar novelty score (\%) provided in the database. An unpaired $t$-test showed that the novelty score for objects in each pair was not significantly different $\left(M_{1}=76.90 \%\right.$, $\left.S D_{1}=11.47 \% ; M_{2}=78.60 \%, S D_{2}=12.04 \%, t(9)=-0.50, p=.627\right)$, suggesting that each pair of the incidental objects chosen was equally novel.

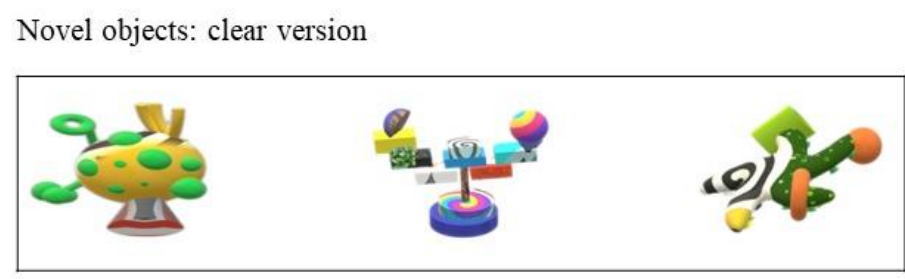

Novel objects: blurred version

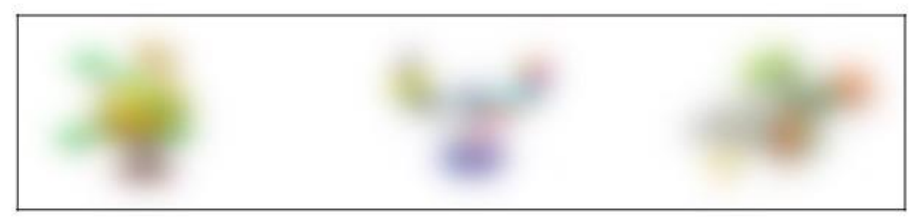

Incidental objects

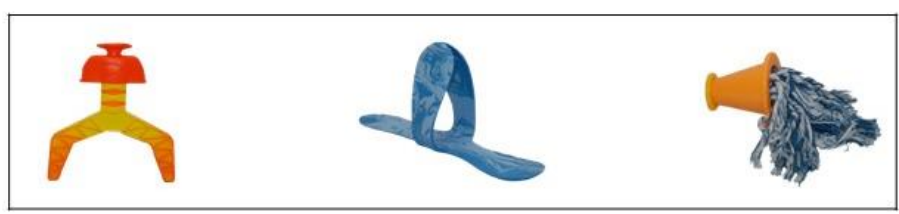

Figure 1. Sample images of stimuli. Top: Clear novel objects; Middle: Blurred, clear, novel objects; Bottom: Incidental novel objects.

\subsubsection{Design}

The experiment consisted of 10 trials, each lasting $34 \mathrm{~s}$ and comprising an exposure phase (22 s) and a preferential looking test phase (12 s). In each exposure phase, infants saw a Curiosity sequence and a Non-Curiosity sequence, counterbalanced across trials and between infants. In the Curiosity sequence, a blurred image was first presented on one side of the screen for $4 \mathrm{~s}$ to induce infants' curiosity, followed by a novel object image as incidental information for $3 \mathrm{~s}$ on the opposite side to the blurred image. Then, the clear corresponding image of the blurred image was presented on the same side as the blurred image for $3 \mathrm{~s}$. The 
Non-Curiosity sequence was similar to the Curiosity sequence except that the first image was always clear and not blurred. At the beginning of and between the two sequences, a central attention getter was presented for $1 \mathrm{~s}$ to maintain attention. The order of novel incidental objects was randomized across sequences and infants.

The exposure phase was followed by a preferential looking test phase in which images of the two incidental objects shown in the exposure phase were presented side by side for $5 \mathrm{~s}$, followed by the same objects on reverse sides for further $5 \mathrm{~s}$ to account for potential orientation bias. The side on which the image from the Curiosity sequence was displayed first was counterbalanced across trials. Figure 2 presents an example of stimulus presentation order.

\section{A Exposure phase}

Curiosity sequence

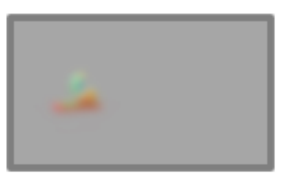

$4 \mathrm{sec}$

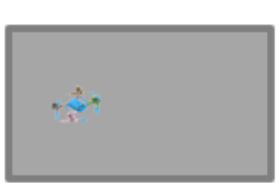

Non-Curiosity sequence

\section{B Preference test phase}

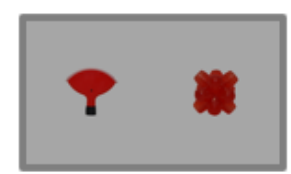

$5 \mathrm{sec}$

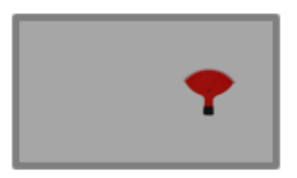

$3 \mathrm{sec}$
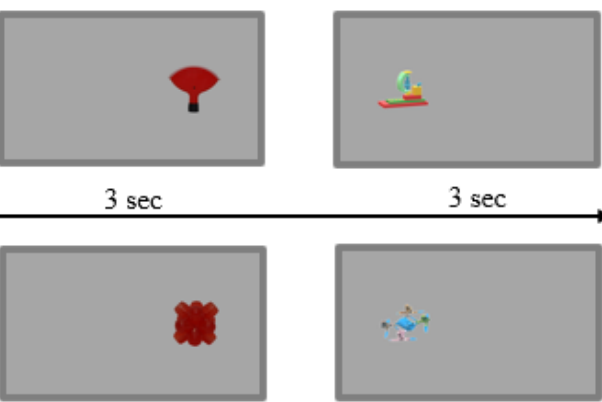

$3 \mathrm{sec}$

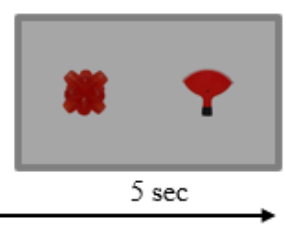

Figure 2 An example of stimulus presentation order in Experiment 1: A) Exposure phase with two counterbalanced sequences (Curiosity and Non-Curiosity sequence) presented sequentially. B) Preferential looking test phase: two incidental objects presented in the learning phase immediately following the exposure phase. 


\subsubsection{Procedure}

Infants sat on their caregiver's lap approximately $60 \mathrm{~cm}$ away from the screen (24inch, resolution 1920 x 1080 pixels) on which the stimuli were presented. A Tobii X120 eye tracker with a gaze sampling rate of $60 \mathrm{~Hz}$ positioned below the screen tracked infants' gaze location and duration. A five-point calibration was conducted before presentation of the stimuli. Recalibrations were conducted if calibration failed. No infants were excluded due to calibration failures. Caregivers were instructed to keep silent and not to influence their infant's behaviour.

\subsubsection{Data processing and cleaning}

Raw eye tracking data were exported from Tobii Studio (Version 3.4) and imported to RStudio (Version 1.1.456) for cleaning and analysis. Rectangular AOIs with a size of $550 \mathrm{x}$ 410 pixels were defined for the left and right AOI for both phases. AOIs were centred on the objects' stationary locations. The margin between the left and the right AOI was 200 pixels. Analysis was conducted from $700 \mathrm{~ms}$, at which point the stimuli stayed stationary.

Data pre-processing was trial-based with 374 trials collected in total. Across all trials, 70 trials were removed due to the eye tracker failing to reliably detect an eye. Trials were excluded when infants looked for less than $100 \mathrm{~ms}$ at each AOI in the exposure phase and the test phase $(\mathrm{n}=41)$. A window size of $80 \mathrm{~ms}$ has been defined as a minimum fixation duration (Wass et al., 2011) in previous research. Considering that infants have slower processing speed, we expanded this window size to $100 \mathrm{~ms}$. Thus, 37 of 39 infants contributed 263 trials for further analysis. Given that only $24.04 \%$ of trials for the second test image pair were valid, only the first pair was analysed in this experiment.

\subsection{Results}

\subsubsection{Exposure phase}


To understand infants' looking behaviors during the exposure phase, we conducted three paired $t$-tests (two tailed) on the mean looking time to each stimulus across the two sequences. First, infants looked significantly longer to the first clear stimulus in the NonCuriosity sequence $(M=2247 \mathrm{~ms}, S D=482 \mathrm{~ms})$ than to the blurred stimulus in the Curiosity sequence $(M=1010 \mathrm{~ms}, S D=428 \mathrm{~ms}, d=2.56 ; t(36)=-15.57, p<.001)$. An estimated Bayes factor of $\mathrm{BF}_{01}<0.01$ using a Cauchy distribution with width of .707 was computed, suggesting very strong evidence for the alternative hypothesis that infants looked longer at the first clear stimulus in the Non-Curiosity sequence (BayesFactor package; Jarosz \& Wiley, 2014; Rouder et al., 2009). Second, there was no evidence for a difference in looking to the two incidental objects in both sequences (Curiosity objects: $M=1532 \mathrm{~ms}, S D=344 \mathrm{~ms}$; Non-Curiosity objects: $M=1594 \mathrm{~ms}, S D=324 \mathrm{~ms}, d=0.19 ; t(36)=-1.14, p=.26)$. The Bayes factor $\mathrm{BF}_{01}$ was 3.11 , which was substantially in favour of the null hypothesis that infants did not preferentially fixate either of the two incidental object images in this exposure phase. Third, there was no significant difference in looking to the final objects in the Curiosity sequence $(M=1583 \mathrm{~ms}, S D=346 \mathrm{~ms})$ and Non-Curiosity sequence $(M=1500 \mathrm{~ms}$, $S D=356 \mathrm{~ms} ; d=0.25 ; t(36)=1.49, p=.14)$. Results from the Bayes Factor analysis $\left(\mathrm{BF}_{01}=\right.$ 2.05) suggested anecdotal evidence for the null hypothesis over the alternative.

\subsubsection{Preference test phase: Total looking preference}

Next, and crucially, to examine the effect of curiosity on the processing of incidental information, we calculated a one-sample $t$-test (two tailed) against chance $(0.5)$ on the proportion looking to each of the two incidental objects on the first test trial. Overall, infants showed a significant preference for the incidental objects presented in the Non-Curiosity sequence $(M=0.56, S D=0.10, d=0.60 ; t(36)=3.58, p=.001$; see Figure 3$)$. A Bayes factor of $\mathrm{BF}_{01}=0.03$ suggested very strong evidence for the alternative hypothesis that infants preferred the incidental objects presented in the Non-Curiosity sequence. We also examined 
changes in proportion looking across the time course of each trial. A bootstrapped clusterbased permutation analysis using the eyetrackingR package (Dink \& Ferguson, 2015) against chance (0.5) was conducted on proportion target looking collapsed into $200 \mathrm{~ms}$ time bins. From $400 \mathrm{~ms}$ to $1200 \mathrm{~ms}$, infants' looking to the Non-Curiosity incidental object was significantly above chance $(p=.02)$, with looking preference not reaching significance after this interval (see Figure 4).

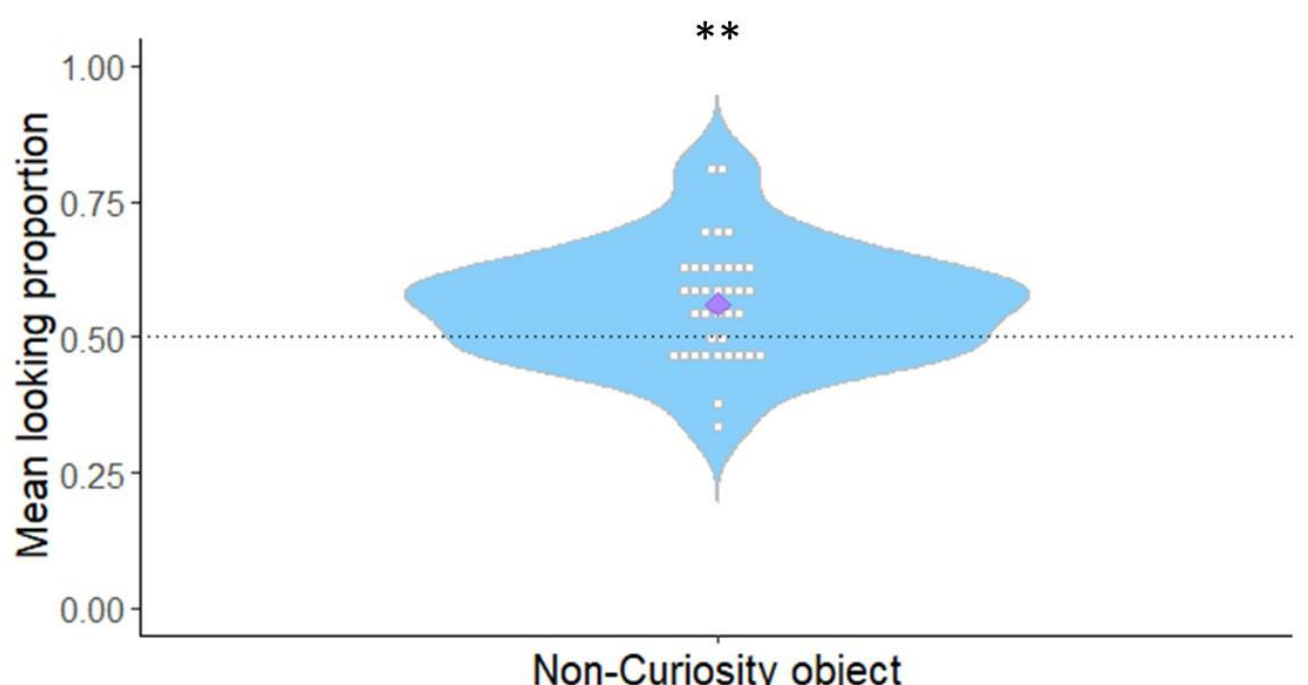

Figure 3. A violin plot of the total proportion looking to the Non-Curiosity object: The purple diamond represents the mean proportion looking time to Non-Curiosity incidental objects. Dashed line represents chance $(0.5) .{ }^{*} p=.001$, two tailed. 


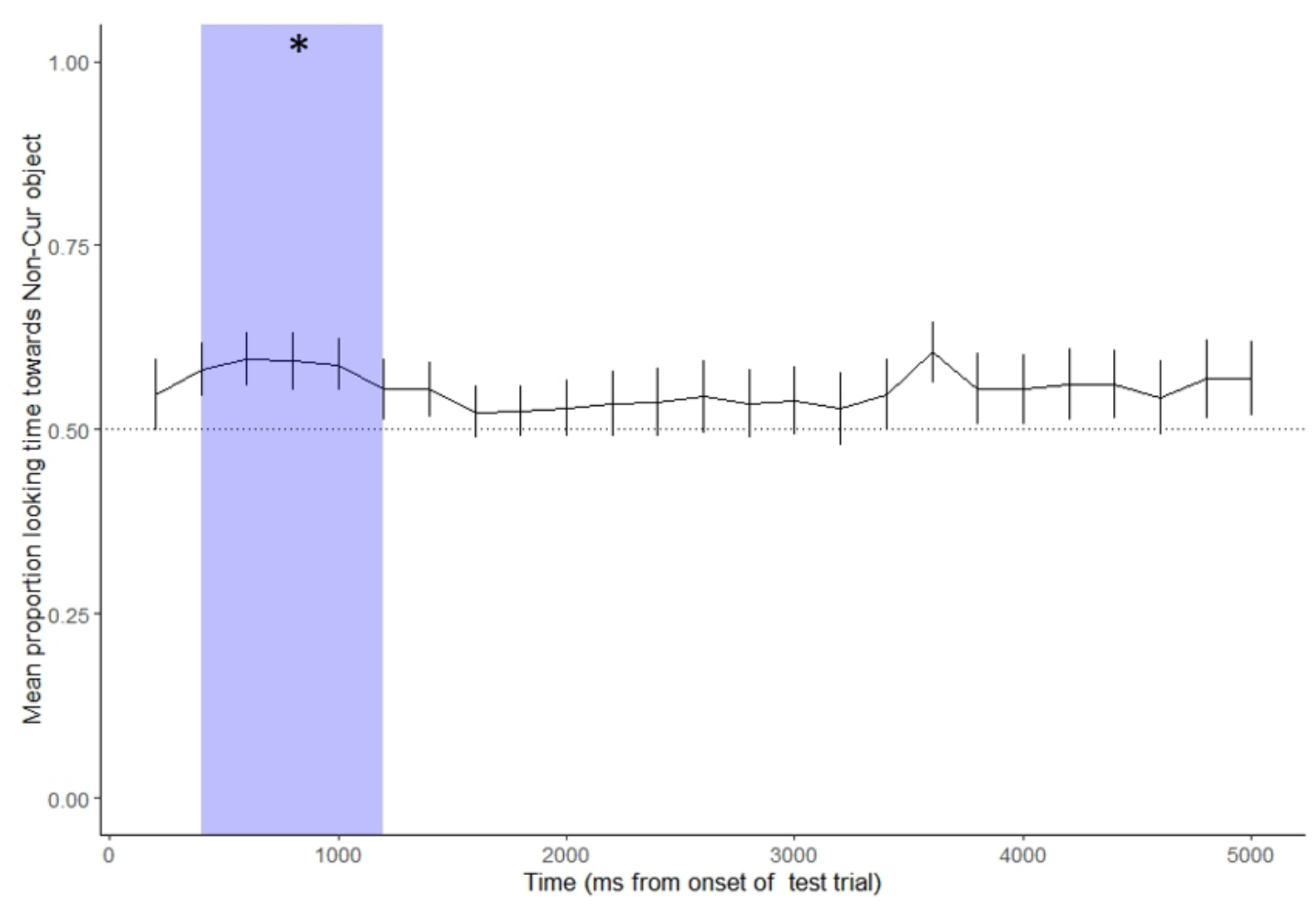

Figure 4. Time course of looking at each of the incidental objects during test: The purple area indicates where the mean proportion looking towards Non-Curiosity objects was above chance (0.5). Dashed line represents chance. ${ }^{*} p<.05$, two tailed.

\subsection{Discussion}

Results from Experiment 1 support the argument that curiosity induced by blurred stimuli enhances infants' processing of subsequent incidental information. Infants spent equal amounts of time looking at the incidental objects in both sequences during the exposure phase. At test, however, infants preferred the Non-Curiosity objects over the Curiosity objects. It is well-known that at this age, patterns of selective looking in infants are likely to be influenced by the novelty of visual stimuli (Ruff \& Rothbart, 2001). A preference for one of the two stimuli is taken to indicate that the other stimulus is more fully processed (Reynolds, 2015). Our findings suggest that the encoding of the incidental objects in the 
Curiosity sequences was more robust than that of objects in the Non-Curiosity sequences, resulting in a novelty preference for the Non-Curiosity item in the later test trials.

As infant looking can indicate both a preference for novelty and familiarity (Hunter \& Ames, 1988), an alternative explanation of our results could be that infants showed a familiarity preference for the Non-Curiosity object, which would suggest that they had processed this object more deeply than the Curiosity object. While we cannot definitely exclude this possibility, we believe it to be unlikely due to the prevalence of novelty preference at this age (Hunter et al., 1983) and evidence showing a persistent decrease in familiarity preference before the age of 6 months (Fisher-Thompson, 2014; Fisher-Thompson \& Peterson, 2004). Hunter and colleagues (1983) examined the effects of familiarization time and complexity of stimuli on infants' familiarity-novelty preference, and found that only when the habituation to a stimulus was interrupted and only when the stimulus was complex, would 8-month-old infants show a familiarity preference. In our study, infants spent an equal amount of looking time at either of the incidental objects with the same level of complexity during familiarization without being interrupted. These findings suggest that in our study infants did show a novelty preference at test.

Another alternative explanation for our results might be that infants did show a novelty preference and had encoded the Curiosity object more deeply, but not because they were in a state of curiosity induced by the blurred image, but because the blurred image had required less processing capacity so that more capacity was left to process the subsequent incidental image. As discussed above, we cannot say with certainty that viewing the blurred image had aroused curiosity in the infants, but adults' self-report of strong curiosity to blurred images and the evidence that perceptual uncertainty elicits exploratory behavior in infants and even in animals, supports this view. Furthermore, the alternative explanation assumes that a fixed amount of attention is available to be distributed across a number of 
subsequent stimuli, but to our knowledge no evidence exists for this theory. To the contrary, infant studies usually contain 'attention getters' to re-orient infants to the screen and 'refresh' their attention for the subsequent experimental stimuli.

We found that infants spent less time looking at the blurred image in the Curiosity sequences compared to the first clear image in the Non-Curiosity sequences. It is well established that infant looking time is driven both by stimulus novelty (i.e., how it relates to infants' prior knowledge) and stimulus complexity (the amount of detail; e.g., Cohen et al., 1975; Fantz \& Nevis, 1967; Hunter et al., 1983). In the current study, the blurred images contained less detail and were less perceptually complex than the clear images, resulting in infants spending less time processing them compared with the clear images. However, a second result of interest in our study is that infants spent equal amounts of time looking at the final images in the Curiosity and Non-Curiosity sequences in the exposure phase, despite the former representing a resolution of curiosity and the latter, not. This result is in contrast with studies in which older children, adolescents and adults showed preferences for resolving curiosity (Fandakova \& Gruber, 2021; Jepma et al., 2012; Nicki, 1970).

In Experiment 2 we aimed to explore whether infants would likewise seek resolution of curiosity without intervening incidental information. Infants first saw a blurred image, followed by a pair of two clear images, one of which was the clear version of the blurred image and the other, a novel image. We tested whether infants would preferentially look at the clear version of the blurred picture over the novel image and thus indicate a preference for the satisfaction of curiosity by uncertainty resolution.

\section{Experiment 2: Resolution of Uncertainty}

\subsection{Method}




\subsubsection{Participants}

The same infants $(N=39)$ who participated in Experiment 1 took part in this experiment.

\subsubsection{Stimuli}

Forty new image stimuli were generated according to the method described in Experiment 1, resulting in 20 blurred images and 20 corresponding clear images. All the stimuli were presented in the form of pictures. No infant saw the same stimulus in more than one trial in this experiment.

\subsubsection{Design}

There were 10 trials, each lasting $7 \mathrm{~s}$. On each trial, a blurred object was presented in the centre of the screen for $3 \mathrm{~s}$ to induce infants' curiosity. Following this, its clear corresponding image paired with another clear, new object were presented side-by-side for 3 s. Infants' eye movements were recorded throughout each trial. The location of the paired clear images was counterbalanced across participants. Figure 5 presents an example of stimulus presentation order.

\subsubsection{Procedure}

The experimental set-up was the same as for Experiment 1.

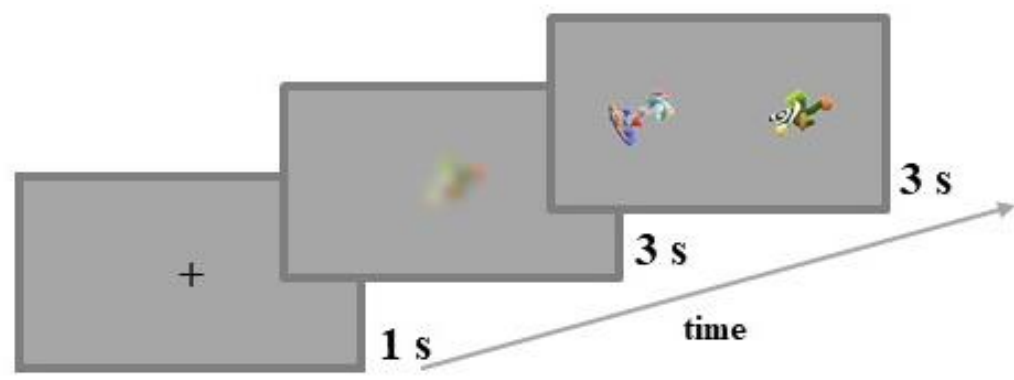

Figure 5. An example of stimulus presentation order in Experiment 2

\subsubsection{Data processing and analysis}


For the central AOI of the blurred images we defined a rectangular AOI of 550 x 410 pixels, and for the left and right AOIs we defined two rectangular 550 x 410 pixel AOIs. The margin between the left and the right AOI was 200 pixels. Data pre-processing was trialbased with 390 trials collected in this experiment. Across all trials, 12 were removed due to the eye tracker failing to reliably detect an eye. We excluded 12 further trials due to experimenter error, and 102 trials with less than 100 ms looking time at each AOI, leaving 39 participants contributing 264 trials in total for further analysis.

\subsection{Results}

Experiment 2 investigated whether infants, after seeing a blurred object, show more interest in the corresponding clear object (target) or in a novel, clear object (distractor).

We submitted proportion target looking (target looking / (target + distractor looking)) to a two-tailed one-sample $t$-test against chance $(0.5)$. Overall, proportion target looking $(M=$ $0.50, S D=0.09, d=0.01)$ was not significantly different from chance $(t(38)=-0.09, p=.93$, Figure 6), indicating no preference for either the target or the distractor object. In order to understand the changes in proportion target looking across the time course of the test trials, we collapsed timestamps into $200 \mathrm{~ms}$ time bins. A bootstrapped cluster-based permutation analysis using the eyetrackingR package (Dink \& Ferguson, 2015) was performed on the averaged target looking proportion in each bin against chance (0.5). Infants' proportion target looking was not different from chance at any point in the trial (see Figure 7). In order to determine whether this result provided evidence for the null hypothesis, we computed an estimated Bayes factor with Cauchy distribution with a width of .707 (the BayesFactor package; Jarosz \& Wiley, 2014; Rouder et al., 2009). This result suggested that the data were 5.77:1 in favour of the null hypothesis that infants did not preferentially fixate either of the two images. 


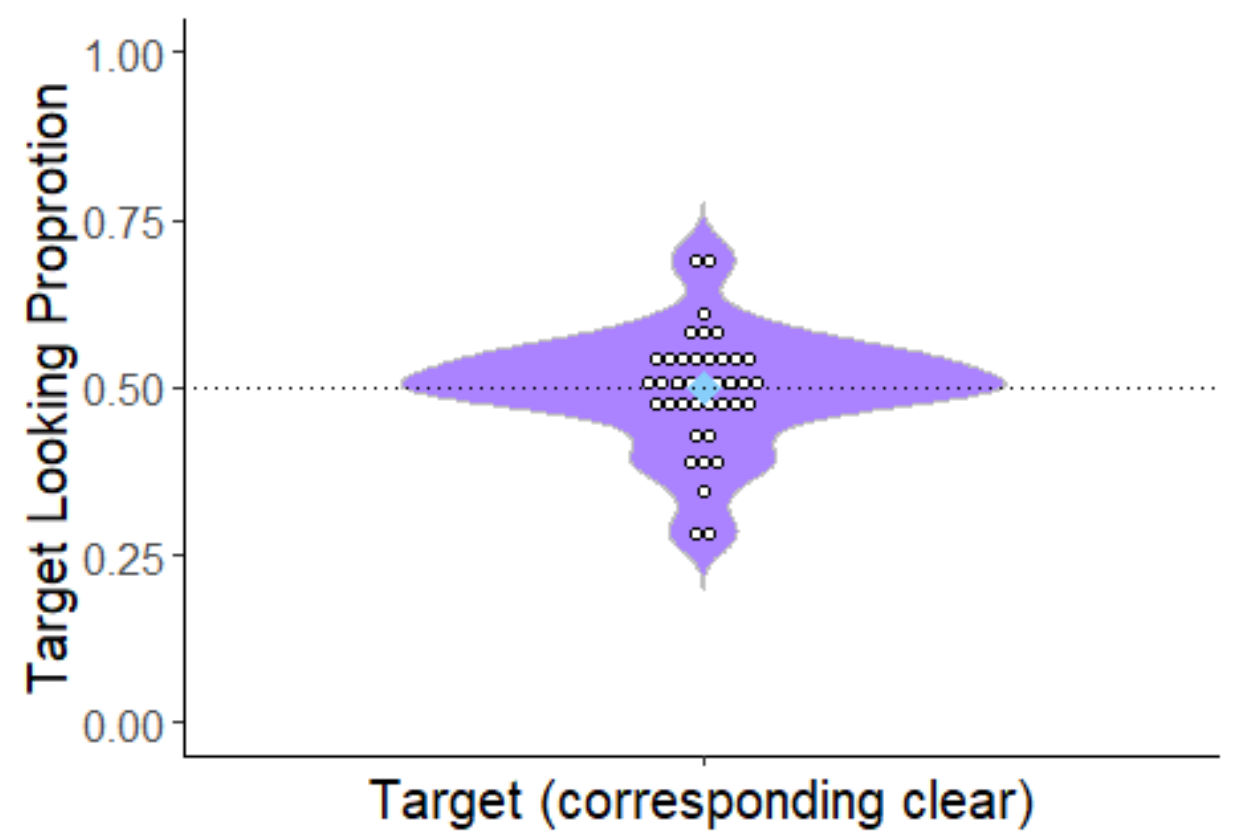

Figure 6. A violin plot of proportion target looking: The central blue dot represents the mean proportion looking time to target objects. Dashed line represents chance (0.5).

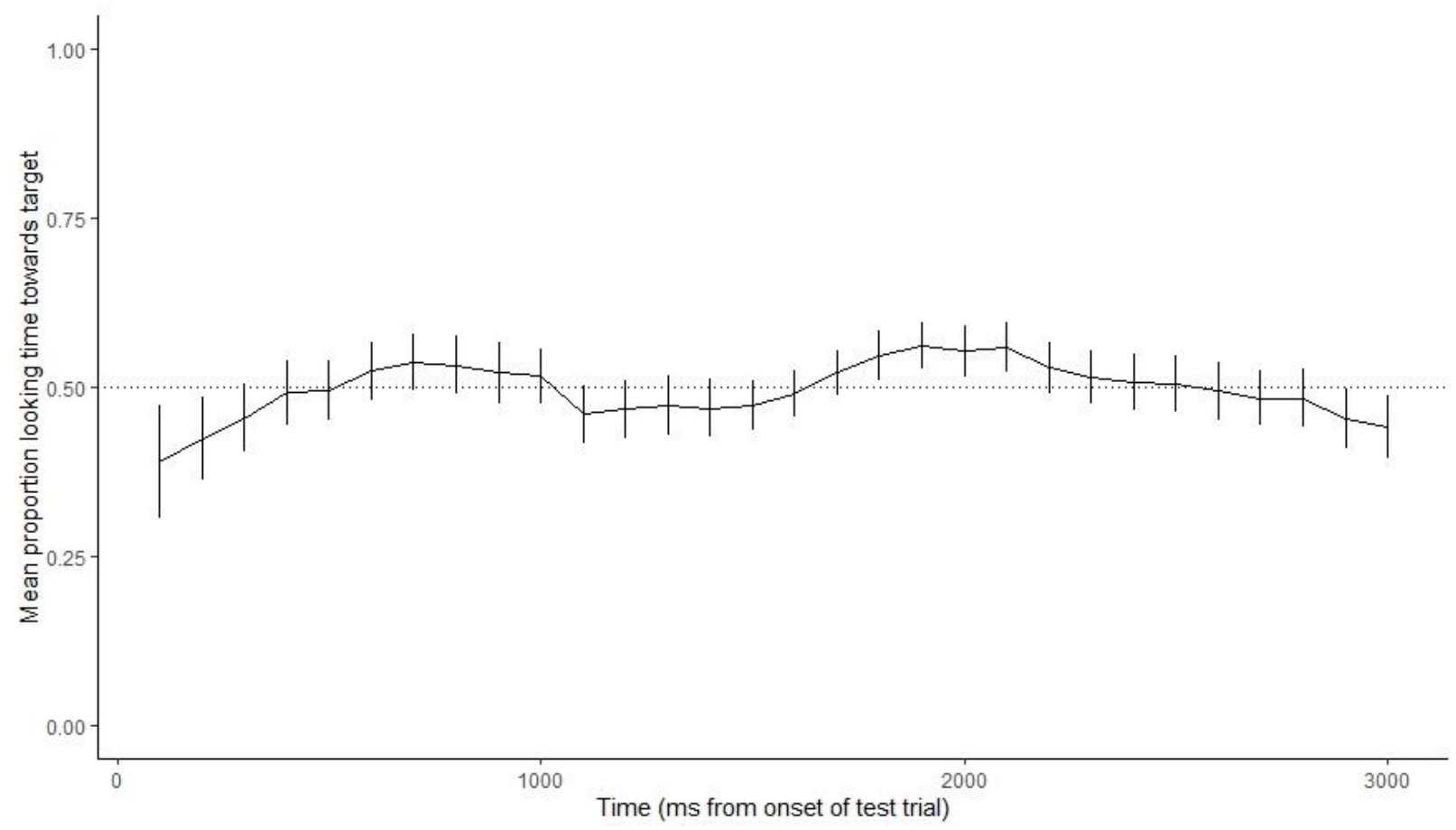

Figure 7. Time course of target looking proportion during test: Infants' proportion target looking was not different from chance (0.5) at any point in the trial. Dashed line represents chance. 


\subsection{Discussion}

Experiment 2 revealed that after seeing a blurred object, infants did not preferentially look at the clear version of this object compared with a new, equally clear object. These results are in conflict with previous studies with adults where participants showed preferences for information that could resolve their curiosity (Nicki, 1970). Therefore, our results provide evidence against a similar preference for resolving curiosity over novelty seeking in infants.

These results suggest that the role of curiosity resolution varies from infants to adults developmentally. In particular, it is possible that the curiosity resolution effect in adults stems from adults' ability to explicitly reason about their curiosity and deliberately attempt to resolve it. Evidence for this assumption comes from the finding that adults are more curious about missing information when they have a hypothesis about what this information is (Wade \& Kidd, 2019). In contrast, young infants are unlikely to be capable of this level of metacognition.

Another possible account of these null findings is the competition between a drive for curiosity resolution and infants' novelty preference. In Experiment 2, infants were not only shown a resolution object but also a new object. Given that infants often show a novelty preference in looking tasks it is possible that the novelty of the new object attracted infants' attention, masking any drive to resolve their curiosity. We note that although in Nicki's (1970) paradigm participants had to choose, through a key press, between revealing the clear version of a blurred image and a new image, they did not see the competing images side-byside. It is possible that in adults too, the presence of a novel competitor would reduce the drive for curiosity resolution. 


\section{General Discussion}

The current studies explored how states of curiosity modulated by visual uncertainty affect object encoding in 8-month-old infants, and whether infants prefer resolution of uncertainty over novelty in curiosity-driven processing. In Experiment 1, we asked whether uncertainty-induced curiosity enhances learning of incidental information. In Experiment 2 we asked whether infants' curiosity induced by blurred images leads them to seek resolution of uncertainty over experiencing novelty. Consistent with previous research with adults (Gruber et al., 2014) but different from similar work with older children (Fandakova \& Gruber, 2021), Experiment 1 suggested that curiosity induced by blurred images indeed enhanced incidental object processing in 8-month-old infants. Contrary to theories of curiosity in the adult literature, however, Experiment 2 found no evidence that infants showed a drive for uncertainty resolution.

Our results suggest that in young infants, curiosity has a broad, attention enhancing effect that is not specific to the object of curiosity. Infants showed enhanced learning for unrelated information encountered while they were in a state of curiosity, and they showed no preference for resolving their curiosity. Taking into account existing studies with older children and adults (Gruber et al., 2014; Fandakova \& Gruber, 2021), these results point to developmental change in the role and function of curiosity. In contrast to infants, in adults curiosity is more focused, and exploration aims to resolve the uncertainty that elicited the curiosity. This strategy is in line with the information gap theory, which postulates that curiosity is triggered by a perceived gap in knowledge (such as the answer to a trivia question, or the identity of a blurred object; Loewenstein, 1994). Perceiving an information gap, however, presupposes metacognitive awareness that is lacking in young infants. 
At the same time, information sampling is also affected by developmental change in working memory (Cowan, 2016), and it is possible that enhanced memory capacity in adults enables them, but not older children, to retain incidental information despite a focus of curiosity on the information triggering the curiosity. In other words, these results raise the possibility of a U-shaped developmental trajectory for curiosity-induced memory enhancement for incidental information, with infants and adults, but not older children, showing this effect. In infants, curiosity might induce a more general, unspecific state of arousal which enhances learning generally but which narrows to more specific goal-directed information seeking in older children and adults. With progressive memory development (Ofen et al., 2012; Cowan, 2016) adults, unlike children, may however become better able to recall incidental information despite the more focused curiosity. Older children's inability to learn incidental information would then occur at a developmental stage in which curiosity is already more focused, but memory is not developed enough to also retain information outside this focus.

Another possible explanation for the discrepancy between our results and those for older children and adults is that the focus of curiosity and the learning of incidental information might vary between different types of information. Whereas in the current study we used blurred images to induce curiosity, the studies testing curiosity-based learning of incidental information with older children and adults have used trivia questions (Fandakova \& Gruber, 2021; Gruber et al., 2014). Both blurred images and trivia questions have been shown to induce epistemic curiosity with a drive to reduce uncertainty about the nature of the blurred object and the answer to the trivia question, respectively. However, in contrast with seeing blurred images, being asked to answer trivia questions explicitly triggers the search for an answer, which could lead to a greater focus on this answer than for clear images following their blurred version. In order to investigate the effect of different types of information, future 
work should replicate the trivia question results from incidental learning in older children and adults with blurred images. Additionally, in these studies the incidental information on which participants were tested was faces whereas in the current study it was novel objects. As faces are processed differently from other information such as objects and words (Inamizu et al., 2020; Martin, 2007) it is not clear how enhanced memory for faces relates to that for other information. Thus, further delineating how the breadth of curiosity-driven learning changes across development is an important avenue for future research, considering the profound role of curiosity in motivation and learning. 


\section{References}

Aslin, R. N., \& Smith, L. B. (1988). Perceptual development. Annual Review of Psychology, 39, 435-473. https://doi.org/10.1146/annurev.ps.39.020188.002251

Berlyne, D. E. (1954). A thoery of human curiosity. British Journal of Psychology. General Section, 45(3), 180-191. https://doi.org/10.1111/j.2044-8295.1954.tb01243.x

Berlyne, D. E. (1960). Conflict, arousal, and curiosity. McGraw-Hill Book Company. https://doi.org/10.1037/11164-000

Berlyne, D. E. (1966). Curiosity and Exploration. Science, 153(3731), 25-33. https:// doi: 10.1126/science.153.3731.25

Bromberg-Martin, E. S., \& Hikosaka, O. (2009). Midbrain dopamine neurons signal preference for advance information about upcoming rewards. Neuron, 63(1), 119. https://doi.org/10.1016/J.NEURON.2009.06.009

Cohen, L. B., DeLoache, J. S., \& Rissman, M. W. (1975). The effect of stimulus complexity on infant visual attention and habituation. Child Development, 46(3), 611. https://doi.org/10.2307/1128557

Cowan, N. (2016). Working Memory Maturation. Perspectives on Psychological Science, 11(2), 239-264. https://doi.org/10.1177/1745691615621279

Daddaoua, N., Lopes, M., \& Gottlieb, J. (2016). Intrinsically motivated oculomotor exploration guided by uncertainty reduction and conditioned reinforcement in nonhuman primates. Scientific Reports 2016 6:1, 6(1), 1-15. https://doi.org/10.1038/srep20202

Dink, J. W., \& Ferguson, B. (2015). eyetrackingR: An R library for eye-tracking data analysis. 
Fandakova, Y., \& Gruber, M. J. (2021). States of curiosity and interest enhance memory differently in adolescents and in children. Developmental Science, 24(1), e13005. https://doi.org/10.1111/DESC.13005

Fantz, R. L., \& Miranda, S. B. (1975). Newborn infant attention to form of contour. Child Development, 46(1), 224-228. https://doi.org/10.2307/1128853

Fantz, R. L., \& Nevis, S. (1967). Pattern preferences and perceptual-cognitive development in early infancy. Merrill-Palmer Quarterly of Behavior and Development. https://www.jstor.org/stable/23082720

Fantz, R. L. (1958). Pattern vision in young infants. The Psychological Record, 8(2), 43-47. https://doi.org/10.1007/BF03393306

Fisher-Thompson, D. (2014). Exploring the emergence of side biases and familiarity-novelty preferences from the real-time dynamics of infant looking. Infancy, 19(3), 227-261. https://doi.org/10.1111/INFA.12051

Fisher-Thompson, D., \& Peterson, J. A. (2004). Infant side biases and familiarity-novelty preferences during a serial paired-comparison task. Infancy, 5(3), 309-340. https://doi.org/10.1207/S15327078IN0503_4

Gruber, M. J., Gelman, B. D., \& Ranganath, C. (2014). States of curiosity modulate hippocampus-dependent learning via the dopaminergic circuit. Neuron, 84(2), 486-496. https://doi.org/10.1016/J.NEURON.2014.08.060

Gruber, M. J., \& Ranganath, C. (2019). How Curiosity Enhances Hippocampus-Dependent Memory: The Prediction, Appraisal, Curiosity, and Exploration (PACE) Framework. Trends in Cognitive Sciences, 23(12), 1014-1025. https://doi.org/10.1016/j.tics.2019.10.003 
Hunter, M. A., \& Ames, E. W. (1984). A multifactor model of the determinants of infant exploratory behavior. Infant Behavior and Development, 7, 175. https://doi.org/10.1016/s0163-6383(84)80237-4

Hunter, M. A., Ames, E. W., \& Koopman, R. (1983). Effects of stimulus complexity and familiarization time on infant preferences for novel and familiar stimuli. Developmental Psychology, 19(3), 338-352. https://doi.org/10.1037/0012-1649.19.3.338

Inamizu, S., Yamada, E., Ogata, K., Uehara, T., Kira, J. ichi, \& Tobimatsu, S. (2020). Neuromagnetic correlates of hemispheric specialization for face and word recognition. Neuroscience Research, 156, 108-116. https://doi.org/10.1016/j.neures.2019.11.006

Jarosz, A. F., \& Wiley, J. (2014). What Are the Odds? A Practical Guide to Computing and Reporting Bayes Factors. The Journal of Problem Solving, 7(1). https://doi.org/10.7771/1932-6246.1167

Jepma, M., Verdonschot, R. G., van Steenbergen, H., Rombouts, S. A. R. B., \& Nieuwenhuis, S. (2012). Neural mechanisms underlying the induction and relief of perceptual curiosity. Frontiers in Behavioral Neuroscience, 6, 5. https://doi.org/10.3389/fnbeh.2012.00005

Kang, M. J., Hsu, M., Krajbich, I. M., Loewenstein, G., McClure, S. M., Wang, J. T., \& Camerer, C. F. (2009). The wick in the candle of learning. Psychological Science, 20(8), 963-974. https://doi.org/10.1111/j.1467-9280.2009.02402.x

Kidd, C., \& Hayden, B. Y. (2015). The Psychology and Neuroscience of Curiosity. Neuron, 88(3), 449-460. https://doi.org/10.1016/j.neuron.2015.09.010

Kobayashi, K., Ravaioli, S., Baranès, A., Woodford, M., \& Gottlieb, J. (2019). Diverse motives for human curiosity. Nature Human Behaviour, 1. https://doi.org/10.1038/s41562-019-0589-3 
Loewenstein, G. (1994). The psychology of curiosity: A review and reinterpretation. Psychological Bulletin, 116(1), 75-98. https://doi.org/10.1037/0033-2909.116.1.75

Martin, A. (2007). The representation of object concepts in the brain. Annual Review of Psychology, 58, 25-45. https://doi.org/10.1146/annurev.psych.57.102904.190143

Nicki, R. M. (1970). The reinforcing effect of uncertainty reduction on a human operant. Canadian Journal of Psychology/Revue Canadienne de Psychologie, 24(6), 389-400. https://doi.org/10.1037/h0082875

Norcia, A. M., \& Tyler, C. W. (1985). Infant VEP acuity measurements: analysis of individual differences and measurement error. Electroencephalography and Clinical Neurophysiology, 61(5), 359-369. https://doi.org/10.1016/0013-4694(85)91026-0

Ofen, N. (2012). The development of neural correlates for memory formation. Neuroscience and Biobehavioral Reviews, 36(7), 1708. https://doi.org/10.1016/J.NEUBIOREV.2012.02.016

Oudeyer, P.-Y., \& Smith, L. B. (2016). How Evolution May Work Through Curiosity-Driven Developmental Process. Topics in Cognitive Science, 8(2), 492-502. https://doi.org/10.1111/tops.12196

Poli, F., Serino, G., Mars, R. B., \& Hunnius, S. (2020). Infants tailor their attention to maximize learning. Science Advances, 6(39), eabb5053. https://doi.org/10.1126/sciadv.abb5053

Quinn, P. C., Eimas, P. D., \& Rosenkrantz, S. L. (1993). Evidence for representations of perceptually similar natural categories by 3-month-old and 4-month-old infants. Perception, 22(4), 463-475. https://doi.org/10.1068/p220463

Quinn, P. C., Eimas, P. D., \& Tarr, M. J. (2001). Perceptual Categorization of Cat and Dog 
Silhouettes by 3- to 4-Month-Old Infants. Journal of Experimental Child Psychology, 79(1), 78-94. https://doi.org/10.1006/JECP.2000.2609

Reynolds, G. D. (2015). Infant visual attention and object recognition. Behavioural Brain Research, 285, 34-43. https://doi.org/10.1016/j.bbr.2015.01.015

Rouder, J. N., Speckman, P. L., Sun, D., Morey, R. D., \& Iverson, G. (2009). Bayesian t tests for accepting and rejecting the null hypothesis. Psychonomic Bulletin \& Review, 16(2), 225-237. https://doi.org/10.3758/pbr.16.2.225

Ruff, H. A., \& Rothbart, M. K. (2001). Attention in early development : themes and variations. Oxford University Press.

Skoczenski, A. M., \& Norcia, A. M. (1998). Neural noise limitations on infant visual sensitivity. Nature, 391(6668), 697-700. https://doi.org/10.1038/35630

Smith, L. B., Jayaraman, S., Clerkin, E., \& Yu, C. (2018). The Developing Infant Creates a Curriculum for Statistical Learning. Trends in Cognitive Sciences, 22(4), 325-336. https://doi.org/10.1016/J.TICS.2018.02.004

Twomey, K. E., \& Westermann, G. (2018). Curiosity-based learning in infants: a neurocomputational approach. Developmental Science. https://doi.org/10.1111/desc.12629

Twomey, K. E., \& Westermann, G. (2019). Building the Foundations of Language. International Handbook of Language Acquisition, 102-114. https://doi.org/10.4324/9781315110622-6

van Lieshout, L. L., Vandenbroucke, A. R., Müller, N. C., Cools, R., \& de Lange, F. P. (2018). Induction and Relief of Curiosity Elicit Parietal and Frontal Activity. The Journal of Neuroscience, 38(10), 2579-2588. https://doi.org/10.1523/jneurosci.2816- 
Wade, S., \& Kidd, C. (2019). The role of prior knowledge and curiosity in learning. Psychonomic Bulletin and Review, 26(4), 1377-1387. https://doi.org/10.3758/s13423019-01598-6

Wass, S., Porayska-Pomsta, K., \& Johnson, M. H. (2011). Training Attentional Control in Infancy. Current Biology, 21(18), 1543-1547. https://doi.org/10.1016/j.cub.2011.08.004 